\title{
Effect of random well-width fluctuations on the exciton optical absorption spectrum in single quantum wells
}

\author{
V. Sa-yakanit \\ Forum for Theoretical Science (FTS), Department of Physics, Faculty of Science, Chulalongkorn University, Bangkok 10330, Thailand \\ Ph. Roussignol \\ Laboratoire de Physique de la Matière Condensée, Ecole Normale Supérieure, 24 rue Lhomond 75231 Paris cedex 05, France
}

\author{
G. Slavcheva \\ Department of Electronics \& Electrical Engineering, Glasgow University, Rankine Building, Oakfield Avenue, \\ Glasgow G12 8QQ, United Kingdom
}

(Received 28 June 1999; revised manuscript received 15 March 2000)

\begin{abstract}
The optical density function is calculated in a single quantum well for two-dimensional excitons moving in a random potential in the interfacial plane generated by fluctuations of the quantum-well thickness. Assuming Gaussian statistics for the random potential distribution, we have applied the path-integral approach and obtained in the adiabatic approximation two asymptotic analytical expressions for the low- and high-energy tails of the optical absorption spectrum. The high-energy tail of the exciton absorption line is also calculated using the perturbation theory. In order to obtain the spectrum across the whole energy range an analytical interpolation formula is found between the asymptotic expressions in the two cases, taking into account the proper normalization of the spectral function. The calculated optical density function is asymmetrically broadened, the magnitude of the peak is reduced, and the maximum is shifted to lower energy in both cases considered, as the disorder increases, in agreement with other theoretical results. Using the fitting parameters to the time-resolved photoluminescence data of Zimmermann [Nuovo Cimento D 17, 1801 (1995)], we find that the path-integral method leads to results for the spectral widths (full widths at half maximum) that are closer to those experimentally observable, as compared with results inferred from the perturbation theory approach. This can be attributed to the additional contribution of the localized exciton states from the Kane band tail in the former method. The effect of varying the correlation length (at a fixed depth of the random potential fluctuations) on the optical density function is also studied.
\end{abstract}

\section{INTRODUCTION}

The effect of in-plane interface disorder on the optical properties of two-dimensional (2D) excitons is currently being studied with renewed interest due to its persistence in both cw and time-resolved experiments. In narrow quantum wells (QW's) the interfacial disorder generates potential fluctuations resulting in band tails in the exciton density of states composed from localized states. Due to the static disorder the optical response of the Wannier excitons in QW's exhibits significant broadening and distinct asymmetry of the exciton line manifested by mixed Lorentz-Gaussian line shapes. ${ }^{1}$ Modification of the exciton radiative lifetime and of the corresponding photoluminescence spectra is also predicted as a consequence of the presence of interfacial disorder and exciton localization. ${ }^{2,3}$ In addition to the influence on the line shape, a substantial Stokes shift between the exciton lines in absorption and photoluminescence spectra is observed at low temperatures ${ }^{4}$ due to interface roughness. These phenomena can be viewed as effects of dephasing, or partial breakdown of temporal coherence. As has been pointed out in Ref. 5, the static disorder by itself induces no dephasing since all scattering processes are elastic. On the other hand, since the disorder produces partial exciton localization (due to the band-tailing phenomenon) this in turn results in inhomogeneous distribution of the exciton energies. The incident pulse excites all oscillators in phase, but the excitons with different energies have different phases and they interfere with each other; this can be termed disorderinduced dephasing.

Disorder can also be considered as the origin of momentum broadening and resonant Rayleigh scattering. ${ }^{6-8}$ The effects of the disorder and the exciton inhomogeneous broadening on time-resolved optical spectra are currently being extensively investigated (see, e.g., Ref. 5). Disorder is responsible for the finite rise time in the time-resolved behavior of secondary radiation (Rayleigh scattering and luminescence). ${ }^{8-11}$ These phenomena imply scattering and partial breakdown of spatial coherence.

The microscopic origin of the interface disorder is related to interface roughness (steps or islands) and atomic interdiffusion, e.g., the cationic exchange in $\mathrm{GaAs} / \mathrm{Al}_{x} \mathrm{Ga}_{1-x} \mathrm{As}$, which occurs for thermodynamical reasons and results in compositional fluctuations. We shall focus our attention on the statistical well-width fluctuations arising from local thickness fluctuations during crystal-growth processes. The Fourier spectrum of the interfacial roughness contains shortand long-wavelength components, the latter related to the atomically smooth growth islands (with lateral dimensions exceeding the exciton Bohr radius) separated by onemonolayer steps that have been experimentally observed in GaAs/ $\mathrm{Al}_{x} \mathrm{Ga}_{1-x} \mathrm{As}$ quantum wells by the cathodoluminescence technique. ${ }^{12}$ The photoluminescence spectrum is very sensitive to the size of the islands. For large enough islands 
splitting of the exciton linewidth is observed (see Ref. 13 and references therein). However, in this paper we shall be interested in the small-island-size regime resulting in rapid interfacial fluctuations.

The classical treatment of exciton motion in a disorder interface potential is unable to explain the inhomogeneous broadening and consequent asymmetry of the exciton lines in 2D. ${ }^{14,15}$ Early attempts at obtaining analytical closed-form descriptions of the exciton spectra in the presence of disorder within the quantum-mechanical theory were restricted to obtaining the spectral density function in one dimension with a specified type of statistical random potential distribution ${ }^{16}$ (e.g., a white-Gaussian-noise potential). Most of the present theoretical studies are based on the solution of Schrödinger's equation for the exciton center-of-mass (c.m.) motion. ${ }^{2,9,17,18}$ Within this approximation the assumption that the disorder does not affect the exciton internal degrees of freedom ${ }^{2,9}$ has been made, which is fulfilled provided that the band-edge fluctuation amplitude along the QW plane is smaller than the exciton confinement energy. The latter represents a reasonable assumption, from the experimental point of view, since it is valid for high-quality samples. Zimmermann ${ }^{9}$ solved the Schrödinger equation for the exciton c.m. motion in a random potential by considering an expansion of the potential fluctuations due to the variation of the well width up to lowest order using the transfer matrix method. The exciton optical density function in a one-dimensional Gaussian random potential (an exciton in a rough quantum wire) has been calculated using Dyson's integral equations for the probability densities. ${ }^{19}$ The calculated spectra exhibit a distinct asymmetry and broadening, with the maximum shifted slightly to lower energies, which gives rise to dephasing. Generally, there are fundamental difficulties in solving the problem in 2D and the results in 1D are usually taken as a model for the asymmetric line shape of 2D QW excitons. However, it is likely that such a simplified approach is not be applicable ${ }^{18}$ in modeling real $2 \mathrm{D}$ exciton spectra.

A quantum-mechanical theory of the influence of disorder on free-particle motion was developed in Ref. 17 using a Green's function expansion with respect to the Fourier transform of the binary correlation function of the random potential fluctuations. An analytical formula was derived for the asymmetrically broadened and shifted spectral function, and numerical calculations were performed for Gaussian fluctuations in 1D and 2D using the linear optical susceptibility. Another numerical method for optical absorption in quantum structures was proposed in Ref. 18. It is based on real-space representation of the Hamiltonian and time-dependent solution of the Schrödinger equation and calculation of the optical susceptibility as an initial-value problem. The method has been applied to excitons on rough interfaces and the calculated spectra show that the $1 \mathrm{D}$ model gives only a very rough approximation to the line shape in $2 \mathrm{D}$.

The effect of inhomogeneous broadening on the exciton absorption line was studied with a semiclassical model in Ref. 5, where the exciton resonance frequency was assumed to have a Gaussian distribution. The absorption line shape of a single QW, within the framework of the linear nonlocal response theory was calculated for both homogeneous and inhomogeneous broadening in $1 \mathrm{D}$, resulting in Gaussian tails of the inhomogeneous broadened spectrum. Since this repre- sents a semiclassical treatment, only symmetric line shapes are produced.

The problem of the coupling of the relative and c.m. motions of the electron-hole pair by the disorder potential has been tackled by means of a Green's function theory approach to the optical response of disordered semiconductors proposed in Ref. 20. As an application calculation of the linear optical properties of a semiconductor QW with interface roughness was performed. Due to the anisotropy of the QW structure, the absorption spectrum depends on the angle of the incident light with respect to the QW plane. In order to account for the simultaneous influence of the propagation and disorder, Maxwell's equations were included in the analysis. The linear susceptibility was obtained from the configuration-averaged-polarization Green's function. The optical absorption spectra both parallel and perpendicular to the QW were calculated. An asymmetric shape and a slight redshift were found for the in-plane $1 s$ excitonic resonance.

In this paper we aim to develop a semianalytical quantum-mechanical description of asymmetric exciton line shapes, line broadening, and low-energy shift of the optical absorption spectrum for $2 \mathrm{D}$ excitons in a $\mathrm{QW}$, taking into account the exciton localization in the random potential fluctuations at the interface. The latter is achieved by applying the Feynman path-integral method for calculation of the band tail and semiclassical exciton density of states. Our approach will mainly follow Ref. 2, where two asymptotic expressions for the optical density function for the high- and low-energy tails of the absorption spectrum were obtained. The high-energy tail of the spectrum calculated in Ref. 2 was obtained using perturbation theory and the low-energy tail by the optimal fluctuation technique, ${ }^{21,22}$ assuming a whiteGaussian-noise correlation function. Analytical interpolation formulas joining the two asymptotic expressions have been found for the optical absorption spectrum in the whole energy range.

In what follows, we shall show that such a semianalytical approach turns out to be more advantageous compared to the pure numerical computations in $2 \mathrm{D},{ }^{18}$ and implies linewidths closer to those experimentally observed. In distinction from the method of Efros and Wetzel ${ }^{2}$ we calculate the optical density function using the general path-integral 2D exciton density of states, assuming a Gaussian random potential distribution and a Gaussian binary correlation function. Our calculations are based on the expression for the 3D electron density of states that was derived by one of us ${ }^{23}$ (V.S.) using the Feynman path-integral method and was further generalized for the $d$-dimensional case in Ref. 24. As limiting cases of the general path-integral expression for the exciton density of states in $2 \mathrm{D}$, we have obtained the low- and highenergy tails of the optical absorption spectrum. Thus, in the high-energy limit, in contrast with Ref. 2, we account for the localized exciton states in the semiclassical Kane band tail, ${ }^{25,26}$ which in turn contribute to the high-energy density of states and high-energy tail of the absorption spectrum. Interpolation between the two asymptotics has been performed for an optical density function depending on two variables (energy and the variance of the random potential), thus obtaining the full 3D profiles of the optical absorption as a function of the disorder (represented by the variance of the random potential fluctuations). Finally, a full 2D exciton 
absorption spectrum calculation is performed. It should be noted that our path-integral approach differs from all other approaches by allowing us to treat on an adequate basis both the short- and long-range correlations in order to account for the whole spectrum of the fluctuating potential.

The outline of the paper is as follows. In Sec. II we describe the theoretical model and the analytical calculations, respectively, for the path-integral approach and the highenergy spectrum tail calculation using the alternative perturbation theory approach. In Sec. III numerical results for the calculated optical absorption spectra are presented, and the influence on the spectra of independently varying the two disorder parameters is studied. Comparison with timeresolved photoluminescence data is also made. Section IV contains concluding remarks.

\section{THEORETICAL MODEL AND ANALYTICAL CALCULATIONS}

\section{A. Optical density function}

We shall consider the motion of a $2 \mathrm{D}$ exciton in a random potential whose wave function according to Ref. 27 can be represented by the product

$$
\Psi\left(\mathbf{r}_{e}, \mathbf{r}_{h}\right)=\Psi(\mathbf{R}) \varphi(\boldsymbol{\rho}) \chi_{e}\left(z_{e}\right) \chi_{h}\left(z_{h}\right),
$$

where $\mathbf{r}_{e, h}\left(\boldsymbol{\rho}_{e, h}, z_{e, h}\right)$ are the electron and hole coordinates, respectively in plane and perpendicular to the plane of a quantum well, $\boldsymbol{\rho}=\boldsymbol{\rho}_{e}-\boldsymbol{\rho}_{h}$ is the coordinate of the relative motion of the two carriers, $\mathbf{R}=\left(m_{e} \boldsymbol{\rho}_{e}+m_{h \|} \boldsymbol{\rho}_{h}\right) / M$ is the exciton's c.m. coordinate, and $M=m_{e}+m_{h \|}$ is the total heavyhole exciton mass, $m_{e}, m_{h \|}$ being the electron and heavyhole masses parallel to the interface. $\Psi(\mathbf{R})$ is the exciton's c.m. wave function describing its motion in the quantumwell plane and $\chi_{e}\left(z_{e}\right)$ and $\chi_{h}\left(z_{h}\right)$ are the wave functions for electron and hole motion in the $z$ direction (perpendicular to the interfacial plane), which for the electrons (or holes) only is that of a particle in a one-dimensional quantum well. $\varphi(\boldsymbol{\rho})$ is the exciton wave function describing the in-plane relative motion of the electron and hole, which in the pure 2D case is given by

$$
\varphi(\boldsymbol{\rho})=\frac{4}{a_{B}^{*} \sqrt{2 \pi}} e^{-2 \rho / a_{B}^{*}},
$$

where $a_{B}^{*}=\epsilon_{s} \hbar^{2} / \mu e^{2}, \quad \epsilon_{s}$ is the semiconductor dielectric constant (assumed equal for both materials of the QW), and $\mu=m_{e} m_{h} / M$ is the reduced effective mass.

Within the adiabatic approximation we shall assume that the exciton line is slightly broadened, i.e., the linewidth is smaller than for the 2D exciton binding energy and the energies of the electron and hole quantization along the $z$ axis within the well. Therefore the solution for the exciton wave function can be factorized out and thus we end up with a Schrödinger equation for a particle of mass $M$ in a random adiabatic potential:

$$
\left[-\frac{\hbar^{2} \nabla^{2}}{2 M}+V(\mathbf{R})\right] \psi(\mathbf{R})=E \psi(\mathbf{R}) .
$$

Our model is aiming to find the spectral function of a single particle (the exciton center of mass) in a field of $N$

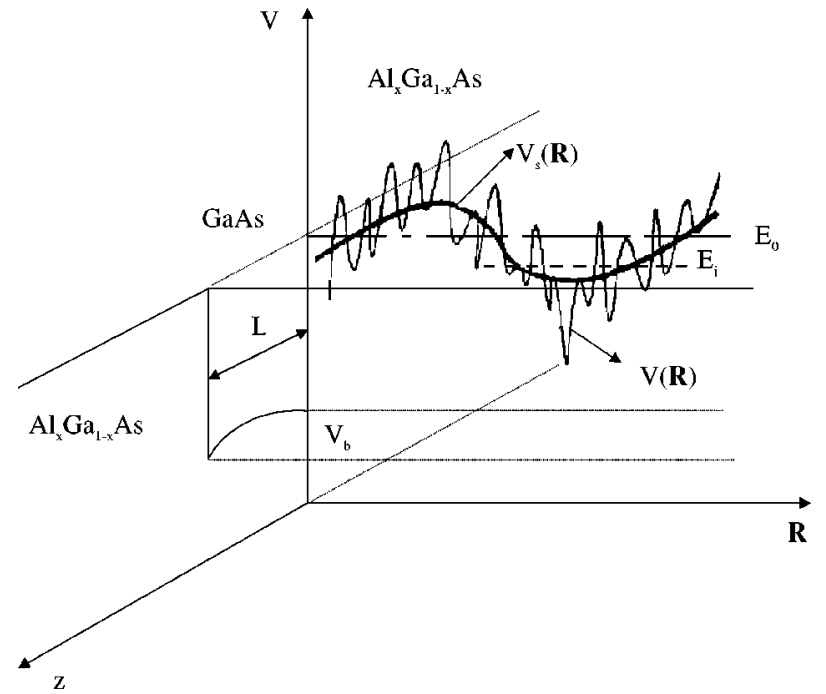

FIG. 1. Plot of the random potential $V(\mathbf{R})$ experienced by the exciton c.m. in the interfacial plane of a single quantum well ( $\mathbf{R}$ is the $2 \mathrm{D}$ in-plane c.m. coordinate) varying about the average potential of the random system $E_{0}$. Along the $z$ axis perpendicular to the interfacial plane the alternating layers of $\mathrm{Al}_{x} \mathrm{Ga}_{1-x} \mathrm{As}$ and $\mathrm{GaAs}$ are represented, showing the potential profile of the quantum well ( $L$ being the width and $V_{b}$ the quantum-well depth). The smoothed potential over the characteristic size of the exciton c.m. envelope wave function (Ref. 29) $V_{s}(\mathbf{R})$ is also represented; $E_{j}$ is a local low-energy level of the exciton c.m., localized in the minimum of the smoothed potential.

scatterers confined in an area $S$ at the interface during its motion near the disordered interface of the quantum well obeying the Hamiltonian

$$
\hat{H}=\hat{H}_{0}+V(\mathbf{R}),
$$

where $\hat{H}_{0}$ is the unperturbed Hamiltonian of the ordered system without randomness and the random in-plane potential $V(\mathbf{R})=\Sigma_{i} v\left(\mathbf{R}-\mathbf{R}_{0 i}\right)$ (Fig. 1) is a superposition of individual scattering potentials $v\left(\mathbf{R}-\mathbf{R}_{0_{i}}\right)$ representing the $2 \mathrm{D}$ random potential generated at a point $\mathbf{R}(x, y)$ in the heterojunction interfacial plane by the local well-width fluctuation located at $\mathbf{R}_{0 i}\left(x_{0 i}, y_{0 i}\right)$ in the plane. The statistical properties of the random potential energy distribution are characterized by its moments. We shall assume a Gaussian statistical distribution, which is completely described by its first and second moments, i.e., by the mean potential energy and the binary correlation function:

$$
\begin{aligned}
& E_{0}=\langle V(\mathbf{R})\rangle=\bar{N}_{2 \mathrm{D}} \int d \mathbf{R}_{0} v\left(\mathbf{R}-\mathbf{R}_{0}\right), \\
& W\left(\mathbf{R}-\mathbf{R}^{\prime}\right)=\left\langle V(\mathbf{R}) V\left(\mathbf{R}^{\prime}\right)\right\rangle \\
&=\bar{N}_{2 \mathrm{D}} \int d \mathbf{R}_{0} v\left(\mathbf{R}-\mathbf{R}_{0}\right) v\left(\mathbf{R}^{\prime}-\mathbf{R}_{0}\right) \\
&=\xi_{L} e^{-\left|\mathbf{R}-\mathbf{R}^{\prime}\right|^{2} / L^{2}},
\end{aligned}
$$

where $\bar{N}_{2 \mathrm{D}}=N / S$ is the surface density of the scattering centers, $L$ is the correlation length of the random potential fluctuations, and $\xi_{L}$ is the variance of the random Gaussian po- 
tential. The quantity $\xi_{L}$, having the dimension of energy squared, was first introduced by Halperin and $\mathrm{Lax}^{29}$ and represents a measure for the depth of the typical potential well. In the $2 \mathrm{D}$ case $\xi_{L}$ is given by

$$
\xi_{L}=\bar{N}_{2 \mathrm{D}}\left(\frac{\pi L^{2}}{4}\right) v_{0}^{2} .
$$

In this expression we have introduced the strength (or amplitude) $v_{0}$ of the individual scattering potentials according to

$$
v\left(\mathbf{R}-\mathbf{R}_{0}\right)=v_{0} e^{-\left|\mathbf{R}-\mathbf{R}_{0}\right|^{2} / l^{2}}
$$

and $L=l \sqrt{2}$.

The spectral density function for a 2D Schrödinger particle in a random potential is defined according to Ref. 16 by

$$
A\left(\mathbf{K}_{\|}, E\right)=\frac{1}{S}\left\langle\sum_{i}\left|\int e^{i \mathbf{K}_{\|} \cdot \mathbf{R}} \Psi_{i}(\mathbf{R}) d \mathbf{R}\right|^{2} \delta\left(E-E_{i}\right)\right\rangle,
$$

where $\Psi_{i}(\mathbf{R})$ is the center-of-mass wave function of the $i$ th exciton state with a corresponding energy $E_{i}, \mathbf{K}_{\|}$is the $2 \mathrm{D}$ exciton wave vector in the plane parallel to the interface, and $S$ is the normalization area. The above averaging is performed over all possible configurations of the random potential fluctuations $(\langle\cdots\rangle$ indicating an average over the statistical ensemble).

Since we shall be interested in the optical absorption spectrum, we wish to calculate the optical density function that represents the $\mathbf{K}_{\|}=\mathbf{0}$ value of the spectral density Eq. (8):

$$
A(E)=\frac{1}{S}\left\langle\sum_{i}\left|\int d \mathbf{R} \Psi_{i}(\mathbf{R})\right|^{2} \delta\left(E-E_{i}\right)\right\rangle .
$$

Then the absorption coefficient can be written as

$$
\alpha(\omega)=\alpha_{0} A(E),
$$

where $\alpha_{0}$ is a slowly varying function of the excitation frequency $\omega$ (see, e.g., Ref. 28).

\section{B. Path-integral approach for calculation of the optical density function}

In order to calculate the optical density function we need to obtain the 2D excitonic density of states (DOS). Reformulating the problem in path-integral (PI) terms, we need to calculate the average (over all random potential configurations) exciton one-particle propagator

$$
\bar{G}\left(\mathbf{R}_{2}, \mathbf{R}_{1} ; t\right)=\int D[\mathbf{R}(\tau)]^{i} e^{i S / \hbar}
$$

satisfying the boundary conditions $\mathbf{R}(0)=\mathbf{R}_{1}, \mathbf{R}(t)=\mathbf{R}_{2}$, where $S$ is the action of the random system, resulting from the averaging over all impurity configurations. $S$ is given up to the second-order moment of the random potential distribution by

$$
S=\int_{0}^{t} d \tau\left(\frac{M}{2} \dot{\mathbf{R}}^{2}(\tau)-E_{0}+\frac{i}{2 \hbar} \int_{0}^{t} d \sigma W[\mathbf{R}(\tau)-\mathbf{R}(\sigma)]\right) .
$$

The density of states per unit area is given by the Fourier transform of the diagonal part of the configurationally averaged one-particle propagator:

$$
\rho(E)=\frac{1}{2 \pi \hbar} \int_{-\infty}^{\infty} d t e^{i E t / \hbar} \bar{G}(0,0 ; t) .
$$

Therefore the problem of a density of states calculation within the framework of the path-integral approach consists in evaluating the exact propagator by integrating over all possible paths using $S$. As was initially pointed out in Ref. 23 , this step can be approximated by introducing a trial action $S_{0}$ based on the harmonic oscillator potential to average the relevant variables over paths. The perturbation $S-S_{0}$ is then calculated using these approximate averages. This procedure is analogous to the use of a "universal wave function" by Halperin and Lax. ${ }^{29}$ The harmonic oscillator potential is equivalent to modeling the real wave function by a quadratic function (Gaussian). The use of a harmonic trial action is equivalent to assuming that all the fluctuating potentials have the same quadratic shape. Thus the problem of the density of states calculation becomes exactly solvable if the full action is approximated by a nonlocal harmonic oscillator "trial" action of the following form:

$$
S_{0}=\int_{0}^{t} d \tau\left(\frac{M}{2} \dot{\mathbf{R}}^{2}(\tau)-\frac{\Omega^{2}}{2 t} \int_{0}^{t} d \sigma|\mathbf{R}(\tau)-\mathbf{R}(\sigma)|^{2}\right) .
$$

The nonlocality of the trial action means that the harmonic oscillator can be anywhere in space. The nonlocal harmonic oscillator frequency $\Omega$ is used as a variational parameter to be adjusted as a function of energy subject to a variational principle.

The average propagator can be rewritten in terms of the trial action, using the path-integral normalization, the trial action introduced above corresponding to a zero-order approximation $\bar{G}_{0}$ to $\bar{G}$. By keeping only the first-order term in the cumulant expansion, a first-cumulant approximation $\bar{G}_{1}$ to $\bar{G}$ is obtained (see Refs. 30 and 31 for details), whose diagonal elements give the density of states (within the firstcumulant approximation).

The DOS per unit volume in the first-cumulant approximation for a Gaussian random potential with a Gaussian binary correlation function was derived in Ref. 23 and generalized in Ref. 24 for the case of a $d$-dimensional system:

$$
\begin{aligned}
\rho_{1}(E)= & \frac{1}{2 \pi \hbar} \int_{-\infty}^{\infty} d t\left(\frac{M}{2 \pi i \hbar t}\right)^{d / 2}\left(\frac{\Omega t}{2 \sin \Omega t / 2}\right)^{d} \\
& \times \exp \left[\frac{d}{2}\left(\frac{\Omega t}{2} \cot \frac{\Omega t}{2}-1\right)\right. \\
& \left.-\frac{1}{2 \hbar^{2}} \xi_{L} t \int_{0}^{t} d x j(x, \Omega)^{-d / 2}+\frac{i}{\hbar}\left(E-E_{0}\right) t\right]
\end{aligned}
$$

where $\Omega$ is a variational parameter to be determined and the function $j(x, \Omega)$ is given by 


$$
\begin{aligned}
j(x, \Omega) & =\left(1+\frac{i \hbar}{M \Omega} \frac{4}{L^{2}} \frac{\sin \Omega x / 2 \sin [\Omega(t-x)] / 2}{\sin Q t / 2}\right) \\
& =\left(1+8 i \frac{E_{L}}{E_{\Omega}} \frac{\sin \Omega x / 2 \sin [\Omega(t-x)] / 2}{\sin \Omega t / 2}\right)
\end{aligned}
$$

where

$$
E_{\Omega}=\hbar \Omega \text { and } E_{L}=\hbar^{2} / 2 M L^{2}
$$

is the correlation energy (the kinetic energy of localization over a distance $L$, the correlation length of the random potential fluctuations), representing a measure of the exciton confinement. It has been previously shown ${ }^{32}$ that in a smalltime approximation the expression for the propagator [equivalent to retaining only high-exciton-energy states in $\rho_{1}(E)$ and physically understood by considering the "pseudo" Heisenberg uncertainty relation $E t \geqslant \hbar$ ] leads to Thomas-Fermi semiclassical results while a large- $t$ approximation reproduces the results of Halperin and $\operatorname{Lax}^{29}$ deep in the band tail.

Two limiting cases of energies in the band tail can be considered. At large negative energies deep in the band tail $\left(E-E_{0} \rightarrow-\infty\right)$, the so-called "quantum case" is valid, for which the following condition is satisfied: $\lambda \gg L$, where $\lambda$ $=h /\left(2 M \sqrt{\xi_{L}}\right)^{1 / 2}$ is the Broglie wavelength of the free exciton and $L$ is the correlation length, i.e., there are no exciton states in a region with the size of the characteristic potential well. The other limiting case $\left(\left|E-E_{0}\right| \rightarrow \infty\right)$ corresponds to a "classical well" containing many exciton states (i.e., $\lambda \ll L$ ). Therefore two analytical asymptotic expressions for the 2D exciton DOS $(d=2)$ can be obtained from the above general expression in the so-called low-energy limit, i.e., deep in the excitonic band tail, and in the opposite extreme of high energy, i.e., near the band edge.

\section{Low-energy limit}

We shall be interested in calculating the optical density function for $\mathbf{K}_{\|}=\mathbf{0}$ in the low-energy tail, i.e., the region of large negative energies below the unperturbed band edge. In what follows we shall apply the path-integral approach for 2D DOS calculations in the presence of a disorder potential ${ }^{23}$ in determining the low-energy tail of the spectral function.

Let us consider first the DOS calculation in the excitonic band tail. As has been discussed in the previous section, in order to evaluate the ground-state energy contribution to the DOS, the limit $t \rightarrow \infty$ of the integrand in the general DOS expression $^{23,33}(15)$ is taken. The 2D exciton DOS deep in the band tail is obtained from that limit, by letting $E \rightarrow$ $-\infty$. Introducing the dimensionless variational parameter $z$ $=E_{\Omega} / E_{L}$ and energy normalizing with respect to the correlation energy according to

$$
\nu=\frac{E_{0}-E}{E_{L}}
$$

the following expression in $2 \mathrm{D}$ is obtained:

$$
\rho_{T}(\nu)=\left[\left(\frac{E_{L}}{L}\right)^{2} / \xi_{L}^{3 / 2}\right] a(\nu, z) e^{-E_{b}^{2}(\nu, z) / 2 \xi_{L}},
$$

where the preexponential factor and the factor in the exponent are obtained analogously to the $3 \mathrm{D}$ derivation $^{33}$ and are given by

$$
a(\nu, z)=\frac{1}{2^{5 / 2} \pi^{3 / 2}} z^{1 / 2}(z+4)^{3 / 2}\left(\frac{z}{2}+\nu\right),
$$

$$
b(\nu, z)=\left(\frac{z}{2}+\nu\right)^{2}\left(1+\frac{4}{z}\right)
$$

In order to obtain the variational parameter $z$ we need to minimize the DOS exponent according to Ref. 29, which in turn leads to the following quadratic equation in the $2 \mathrm{D}$ case considered:

$$
z^{2}+2 z-4 \nu=0
$$

Keeping only the positive root

$$
z=\sqrt{1+4 \nu}-1,
$$

which has physical meaning, and substituting it into Eqs. (20) and (21), we obtain

$$
\begin{aligned}
& a(\nu)=\frac{(\sqrt{1+4 \nu}-1)^{3 / 2}(\sqrt{1+4 \nu}+3)^{5 / 2}}{2^{9 / 2} \pi^{3 / 2}}, \\
& b(\nu)=\frac{1}{2^{4}}(\sqrt{1+4 \nu}-1)(\sqrt{1+4 \nu}+3)^{3} .
\end{aligned}
$$

Introducing the dimenensionless quantity

$$
\xi^{\prime}=\frac{\xi_{L}}{E_{L}^{2}},
$$

we obtain for the 2D DOS deep in the excitonic band tail

$$
\rho_{T}(\nu)=\left(\frac{1}{E_{L} L^{2}} \frac{1}{\xi^{\prime 3 / 2}}\right) a(\nu) e^{-b(\nu) / 2 \xi^{\prime}} .
$$

In order to calculate the optical density function, we rewrite Eq. (9) in the form of a functional integral over all possible potential fluctuations:

$$
A(E)=\frac{1}{S} \int D V W(V) \sum_{i}\left|\int d \mathbf{R} \Psi_{i}(\mathbf{R})\right|^{2} \delta\left(E-E_{i}\right),
$$

where $W(V)$ is the probability of the random potential distribution. The excitonic states deep in the low-energy tail are produced by deep and narrow random potential fluctuations and therefore the energy distance between the ground state and the excited states of the well is greater. Therefore the contribution of the excited states can be neglected deep in the band tail. In the low-energy range $\left(\left(E-E_{0}\right) / E_{L} \rightarrow-\infty\right.$, or, equivalently, $\nu \gg 1$ ) we shall be interested only in the ground-state contribution to the density of states (since we have taken the limit $t \rightarrow \infty$ in obtaining it), and we can consider that the main contribution to the above integral comes from the ground-state exciton center-of-mass wave function in a harmonic potential well, i.e.,

$$
\Psi_{0}(\mathbf{R})=\left(\frac{2 \gamma}{\pi}\right)^{1 / 2} e^{-\gamma R^{2}}
$$


where

$$
\gamma=\frac{M \Omega}{\hbar}=\frac{z}{2 L^{2}}
$$

and $z$ is a variational parameter, the same as that appearing in the band-tail DOS, and is given by Eq. (23). Therefore we can take the factor $\left|\int d \mathbf{R} \Psi_{0}(\mathbf{R})\right|^{2}$ out of the functional integral. The remaining functional integral is, by definition, the band-tail density of states $\rho_{T}(\nu)$. Therefore

$$
A(E)=\left|\int d \mathbf{R} \Psi_{0}(\mathbf{R})\right|^{2} \rho_{T}(E)=\left(\frac{2 \pi}{\gamma}\right) \rho_{T}(E) .
$$

Substituting $\gamma$ and $z$ from Eqs. (30) and Eq. (23) we obtain the following expression for the low-energy tail of the optical density function:

$$
A(\nu)=\frac{4 \pi L^{2}}{(\sqrt{1+4 \nu}-1)} \rho_{T}(\nu) .
$$

\section{High-energy limit}

Let us consider now the calculation of the high-energy tail of the optical density function. In order to obtain the highenergy semiclassical Kane limit of the general PI expression for the 2D exciton DOS near the band edge, it is necessary to take the limit $t \rightarrow 0$ of the integrand of Eq. (15), which corresponds to retaining only high-energy excitonic states in the DOS. Taking into account that $\lim _{t \rightarrow 0} j(x, \omega)=1$, after integration over $t$ this gives

$$
\rho_{K}(E)=\frac{1}{2^{5 / 2} \pi^{3 / 2} E_{L} L^{2}} e^{\left(E_{0}-E\right)^{2} / 4 \xi_{L} D_{-1}}\left(\frac{E_{0}-E}{\sqrt{\xi_{L}}}\right),
$$

where $D_{-1}$ is the parabolic cylinder function of order -1 . Finally, introducing dimensionless variables $\xi^{\prime}$ and $\nu$ we get

$$
\rho_{K}(\nu)=\frac{1}{2^{5 / 2} \pi^{3 / 2} E_{L} L^{2}} e^{-\nu^{2} / 4 \xi^{\prime}} D_{-1}\left(\frac{\nu}{\sqrt{\xi^{\prime}}}\right) .
$$

In the opposite extreme, at high enough energies, the problem of finding the proper variational exciton wave function is not a straightforward one, since the high-energy states are described by noncompact fractal-shaped wave functions ${ }^{38}$ and the corresponding optical absorption spectrum results from many small contributions due to those states. However, it is clear that the exciton c.m. wave functions $\Psi_{i}(\mathbf{R})$ have to be close to plane waves. According to Ref. 34, the exciton states close to the delocalized states can be described quite satisfactorily by choosing a c.m. envelope wave function of the form

$$
\Psi_{i}(\mathbf{R})=A_{0} e^{i \mathbf{K}_{\|} \cdot \mathbf{R}-\gamma \mathbf{R}^{2}}, \quad A_{0}=\left(\frac{2 \gamma}{\pi}\right)^{1 / 2} .
$$

We shall consider that the main contribution to the configurational average in Eq. (9) at high energies is given by states with an equal shape of the envelope wave function, namely, Eq. (35). In what follows, we shall show that this particular choice of the envelope wave function results in realistic optical absorption spectra.
As in the first case (deep in the band tail) we can take the matrix element out of the ensemble averaging and the remaining average gives the semiclassical Kane 2D exciton DOS, Eq. (33).

The matrix element can be directly calculated, yielding

$$
\left|\int d \mathbf{R} \Psi_{i}(\mathbf{R})\right|^{2}=\left(\frac{2 \pi}{\gamma}\right) e^{-\mathbf{K}_{\|}^{4} / 16 \gamma^{2}} .
$$

Therefore

$$
A(E)=\left(\frac{2 \pi}{\gamma}\right) e^{-\mathbf{K}_{\|}^{4} / 16 \gamma^{2}} \rho_{K}(E) .
$$

Substituting $\mathbf{K}_{\|}^{2}=2 M\left(E_{\mathbf{K}_{\|}}-E_{0}\right) / \hbar^{2}$ and introducing the dimensionless energy according to Eq. (18), we obtain

$$
A(\nu)=\left(\frac{2 \pi}{\gamma}\right) e^{-\nu^{2} / 16 \gamma^{2} L^{4}} \rho_{K}(\nu),
$$

where the semiclassical 2D exciton DOS is given by Eq. (29).

Finally, we obtain the following general expression for the optical density function at high energies:

$$
\begin{aligned}
A(\nu)= & \frac{1}{2^{3 / 2} \sqrt{\pi}} \frac{1}{\gamma L^{2} E_{L}} \\
& \times \exp \left[-\nu^{2}\left(\frac{1}{\left(4 \gamma L^{2}\right)}+\frac{1}{4 \xi^{\prime}}\right)\right] D_{-1}\left(\frac{\nu}{\sqrt{\xi^{\prime}}}\right) .
\end{aligned}
$$

In order to obtain the semiclassical Kane band tail we take the limit $\left|E-E_{0}\right| \rightarrow \infty$, i.e., $\left(E-E_{0}\right) / \sqrt{\xi_{L}} \ll-1$ or $\nu / \sqrt{\xi^{\prime}}$ $\gg 1$, and taking into account the asymptotic behavior of the parabolic cylinder function for large argument values, namely, $\lim _{z \rightarrow \infty} D_{p}(z) \sim e^{-z^{2} / 4} z^{p}$, we obtain

$$
A(\nu)=\frac{1}{2 \sqrt{2 \pi}} \frac{\sqrt{\xi^{\prime}}}{\gamma L^{2}} \frac{1}{\nu E_{L}} \exp \left[-\nu^{2}\left(\frac{1}{\left(4 \gamma L^{2}\right)^{2}}+\frac{1}{2 \xi^{\prime}}\right)\right] .
$$

The opposite extreme, $E-E_{0}>0, E-E_{0} \rightarrow \infty$, i.e., ( $E$ $\left.-E_{0}\right) / \sqrt{\xi_{L}} \gg 1, \nu / \sqrt{\xi^{\prime}} \ll 1$, yields the free-exciton (continuum exciton states) 2D DOS (see, e.g., Ref. 35)

$$
\rho^{s c}(\nu)=\frac{M}{2 \pi \hbar^{2}}\left[1+\operatorname{erf}\left(\frac{\nu}{\sqrt{\xi^{\prime}}}\right)\right] .
$$

Therefore, the optical density function acquires the form

$$
A(\nu)=\frac{1}{2 \gamma L^{2} E_{L}}\left[1+\operatorname{erf}\left(\frac{\nu}{\sqrt{\xi^{\prime}}}\right)\right] e^{-\nu^{2} /\left(4 \gamma L^{2}\right)^{2}} .
$$

Finally, we obtain the following two limiting cases: 


$$
A(\nu)=\left\{\begin{array}{l}
\frac{1}{2 \sqrt{2 \pi}} \frac{\sqrt{\xi^{\prime}}}{\gamma L^{2} E_{L}} \frac{1}{\nu} \exp \left[-\nu^{2}\left(\frac{1}{\left(4 \gamma L^{2}\right)^{2}}+\frac{1}{2 \xi^{\prime}}\right)\right], \frac{\nu}{\sqrt{\xi^{\prime}}} \gg 1 \\
\frac{1}{2 \gamma L^{2} E_{L}}\left[1+\operatorname{erf}\left(\frac{\nu}{\sqrt{\xi^{\prime}}}\right)\right] e^{-\nu^{2} /\left(4 \gamma L^{2}\right)^{2}}, \quad \frac{\nu}{\sqrt{\xi^{\prime}}} \ll 1 .
\end{array}\right.
$$

If we take the limit $\nu \rightarrow \infty$ of the free-exciton DOS [second line of Eq. (43)], since for the free excitons the difference between the energy of the exciton c.m. and the mean potential energy $E_{0}$ is expected to be much larger than the localization energy $E_{L}$, we obtain a pure Gaussian exciton line shape:

$$
A(\nu)=\frac{1}{\gamma L^{2} E_{L}} e^{-\nu^{2} /\left(4 \gamma L^{2}\right)} .
$$

The dimensionless parameter $\gamma L^{2}=z / 2$ in Eq. (43) depends on the variational parameter $z$ introduced in the calculation procedure for the low-energy tail of the spectral density and is given by Eq. (23).

A1

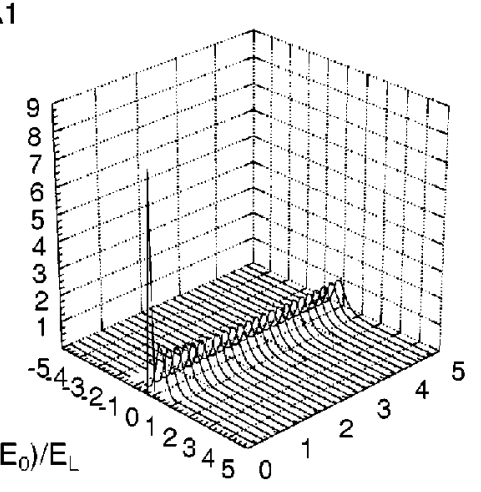

(a)

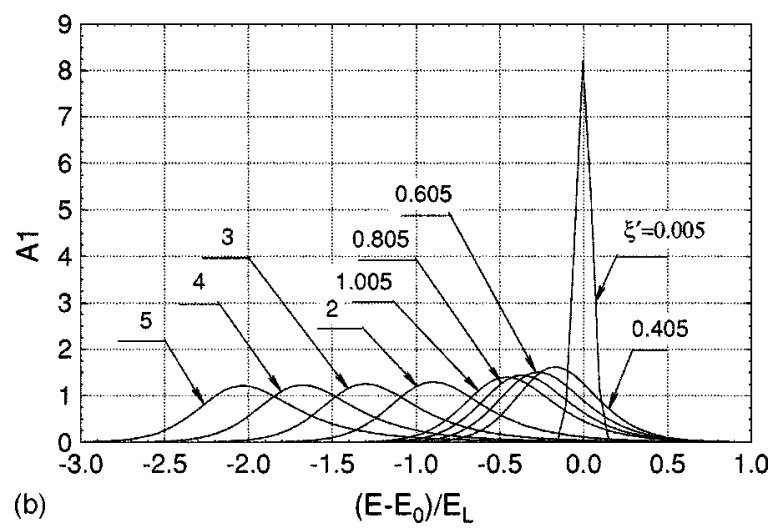

FIG. 2. Plot of the normalized optical density function vs dimensionless energy $\left(E-E_{0}\right) / E_{L}$ and dimensionless disorder parameter $\xi^{\prime}$ calculated on the basis of the perturbation theory high- and low-energy tails, inferred from the path-integral approach (referred to as case 1 in the text). (a) 3D trace plot; (b) cross sections of (a) at $\xi^{\prime}=0.005,0.405,0.605,0.805,1.005,2,3,4,5$. The correlation energy has been kept constant at $E_{L}=0.12 \mathrm{meV}$.

\section{Perturbation theory approach for high-energy tail of the optical density function}

One might expect that the high-energy behavior of the optical absorption spectrum at very weak disorder would be limited by the perturbation theory results accounting for delocalized states in the spectrum. In order to check this, let us calculate the high-energy tail of $A(E)$ using perturbation theory. In this region the exciton center-of-mass envelope wave functions are nearly plane waves.

Using the perturbation theory in the high-energy limit, we have

$A(E)=\frac{1}{S} \int \frac{d^{2} K_{\|}}{(2 \pi)^{2}} \frac{\left\langle\left|\int V(\mathbf{R}) e^{i \mathbf{K}_{\|} \cdot \mathbf{R}} d \mathbf{R}\right|^{2}\right\rangle}{E_{K_{\|}}^{2}} \delta\left(E-E_{K_{\|}}\right)$.

Let us first calculate the average:

$$
\begin{aligned}
\left\langle\left|\int V(\mathbf{R}) e^{i \mathbf{K}_{\|} \cdot \mathbf{R}} d \mathbf{R}\right|^{2}\right\rangle= & \int d \mathbf{R} \int d \mathbf{R}^{\prime} e^{i \mathbf{K}_{\| \cdot} \cdot\left(\mathbf{R}-\mathbf{R}^{\prime}\right)} \\
& \times\left\langle V(\mathbf{R}) V\left(\mathbf{R}^{\prime}\right)\right\rangle .
\end{aligned}
$$

Substituting the correlation function $\left\langle V(\mathbf{R}) V\left(\mathbf{R}^{\prime}\right)\right\rangle$ from Eq. (5) and performing the integration by introducing the new variable $\mathbf{x}=\mathbf{R}-\mathbf{R}^{\prime}$ (because of the translational invariance in the plane parallel to the interface), we obtain

$$
\left\langle\left|\int V(\mathbf{R}) e^{i \mathbf{K}_{\|} \cdot \mathbf{R}} d \mathbf{R}\right|^{2}\right\rangle=\xi_{L} S \pi L^{2} e^{-K_{\|}^{2} L^{2} / 4} .
$$

After performing the integration over the $2 \mathrm{D}$ in-plane wave vector in Eq. (45), finally we obtain the following expression:

$$
A(E)=\frac{\xi_{L} M L^{2}}{2 \hbar^{2}} \frac{e^{-\left(2 M L^{2} / 4 \hbar^{2}\right) E}}{E^{2}} .
$$

Introducing the dimensionless energy according to Eq. (18) and the dimensionless parameter $\xi^{\prime}$ according to Eq. (26) we can rewrite it as

$$
A(\nu)=\frac{\xi^{\prime}}{4 E_{L} \nu^{2}} e^{-\nu / 4}
$$

Comparing Eq. (49) with the path-integral expression (44) at high energies, we can conclude that (for strong enough disorder) the perturbation theory result for the high-energy shoulder of the spectrum decays faster than the semiclassical limit of the path-integral result, resulting in a broader spectrum. As will be shown in Sec. III, for weak disorder the high-energy side of the spectrum tends to the perturbation theory results, while on increasing the disorder the departure of the path-integral result from that of the perturbation theory becomes increasingly pronounced. 
A2

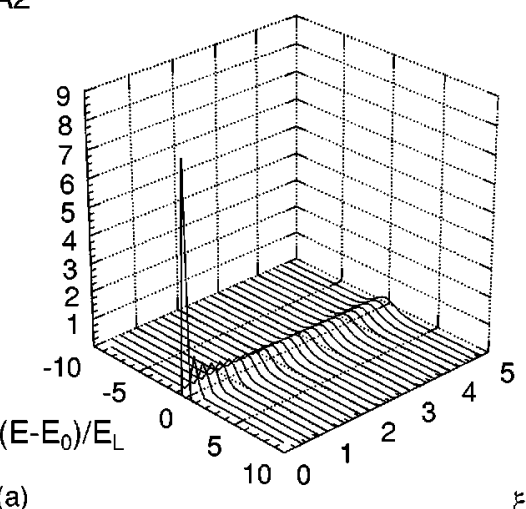

(a)

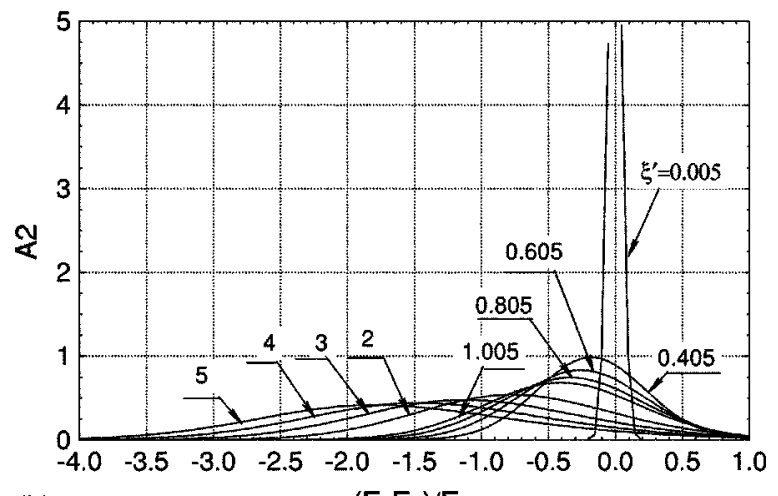

(b)

$\left(\mathrm{E}-\mathrm{E}_{0}\right) / \mathrm{E}_{\mathrm{L}}$

FIG. 3. Plot of the normalized optical density function vs dimensionless energy $\left(E-E_{0}\right) / E_{L}$ and dimensionless disorder parameter $\xi^{\prime}$ calculated using the path-integral method for both the highand low-energy tails of the spectrum (referred to as case 2 in the text). (a) 3D trace plot; (b) cross sections of (a) at $\xi^{\prime}$ $=0.005,0.405,0.605,0.805,1.005,2,3,4,5$. The correlation energy has been kept constant at $E_{L}=0.12 \mathrm{meV}$.

\section{NUMERICAL COMPUTATIONS AND RESULTS}

In Sec. II we have obtained using a path-integral method low-energy [given by Eqs. (32), (27), (24), and (25)], and high-energy [Eq. (39)] asymptotic expressions for the optical density function and an alternative high-energy asymptotics resulting from the perturbation theory [Eq. (49)]. Since we are interested in the spectrum across the whole energy range, an interpolation function between Eqs. (32) and (49), hereinafter referred to as case 1, and also between Eqs. (32) and Eq. (39), referred to as case 2, has to be sought. The interpolation function in both cases has been found by performing a nonlinear fitting procedure based on the $\chi^{2}$ minimization

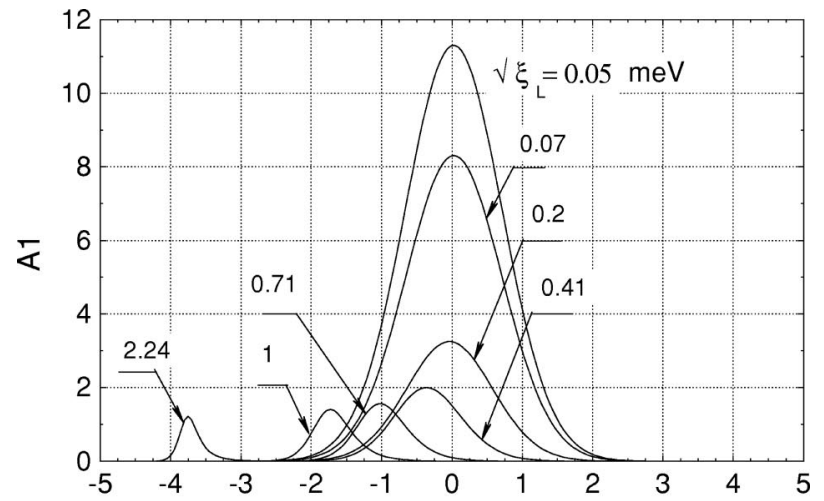

(a)

$\left(\mathrm{E}-\mathrm{E}_{0}\right) / \sqrt{ } \xi_{\mathrm{L}}$

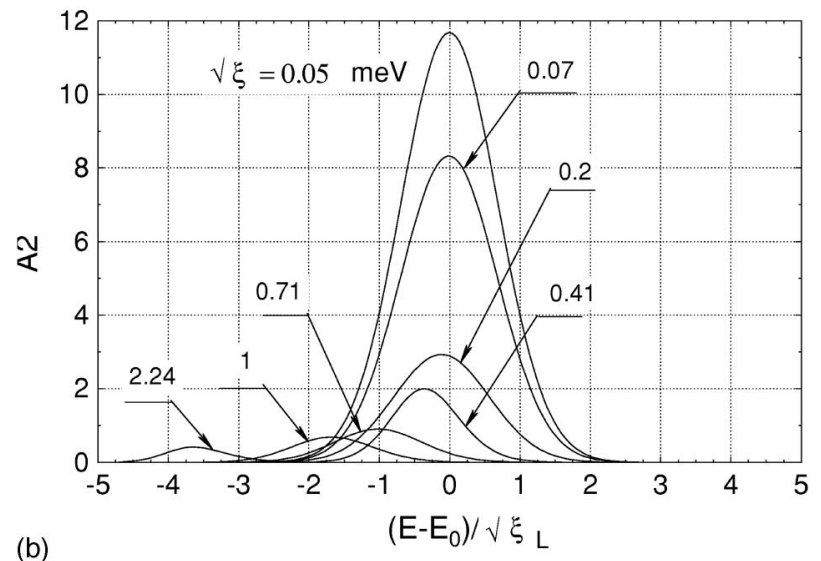

FIG. 4. (a) Plot of the optical density function corresponding to case 1 vs energy, normalized with respect to the standard deviation of the random potential $\sqrt{\xi_{L}}$ : a measure for the magnitude of the potential fluctuations for different values of the standard deviation $\sqrt{\xi_{L}}=0.05,0.07,0.2,0.41,0.71,1,2.24 \mathrm{meV}$; the low-energy shift of the maximum is clearly seen. The plots are calculated assuming a constant correlation energy $E_{L}=0.12 \mathrm{meV}$. (b) Plot of the optical density function corresponding to case 2 vs energy, normalized with respect to the standard deviation of the random potential $\sqrt{\xi_{L}}$ for different values of the standard deviation $\sqrt{\xi_{L}}$ $=0.05,0.07,0.2,0.41,0.71,1,2.24 \mathrm{meV}$ (all the plots are at $E_{L}$ $=0.12 \mathrm{meV})$.

criterion. Using the proper normalization condition for the optical density function fulfilled for any value of the disorder parameter $\xi^{\prime}$,

$$
\int_{-\infty}^{\infty} A\left(\nu, \xi^{\prime}\right) d \nu=1
$$

we obtain the following normalized interpolation functions:

$$
A\left(\nu, \xi^{\prime}\right)=\left\{\begin{array}{l}
\frac{\xi^{\prime} e^{-1.03975 \nu / \xi^{\prime}}\left(0.53665 \nu^{2}+1.35851 \nu+0.81927\right)}{\left(\nu^{2}+0.28551\right)^{2}\left[4.21614 \sqrt{\xi^{\prime}}+10.01300 e^{0.296863 / \xi^{\prime}}\left(\xi^{\prime}-0.40664\right) \operatorname{erfc}\left(\frac{0.54485}{\sqrt{\xi^{\prime}}}\right)\right]}, \quad \text { case 1 } \\
\frac{0.23810 e^{-2.83076 / \xi^{\prime}}}{\operatorname{erfc}\left(\frac{1.68249}{\sqrt{\xi^{\prime}}}\right)} \frac{e^{-1.0650 \nu^{2} / \xi^{\prime}}(\nu+2.18072)}{\nu^{2}+2.66678}, \quad \text { case 2 }
\end{array}\right.
$$



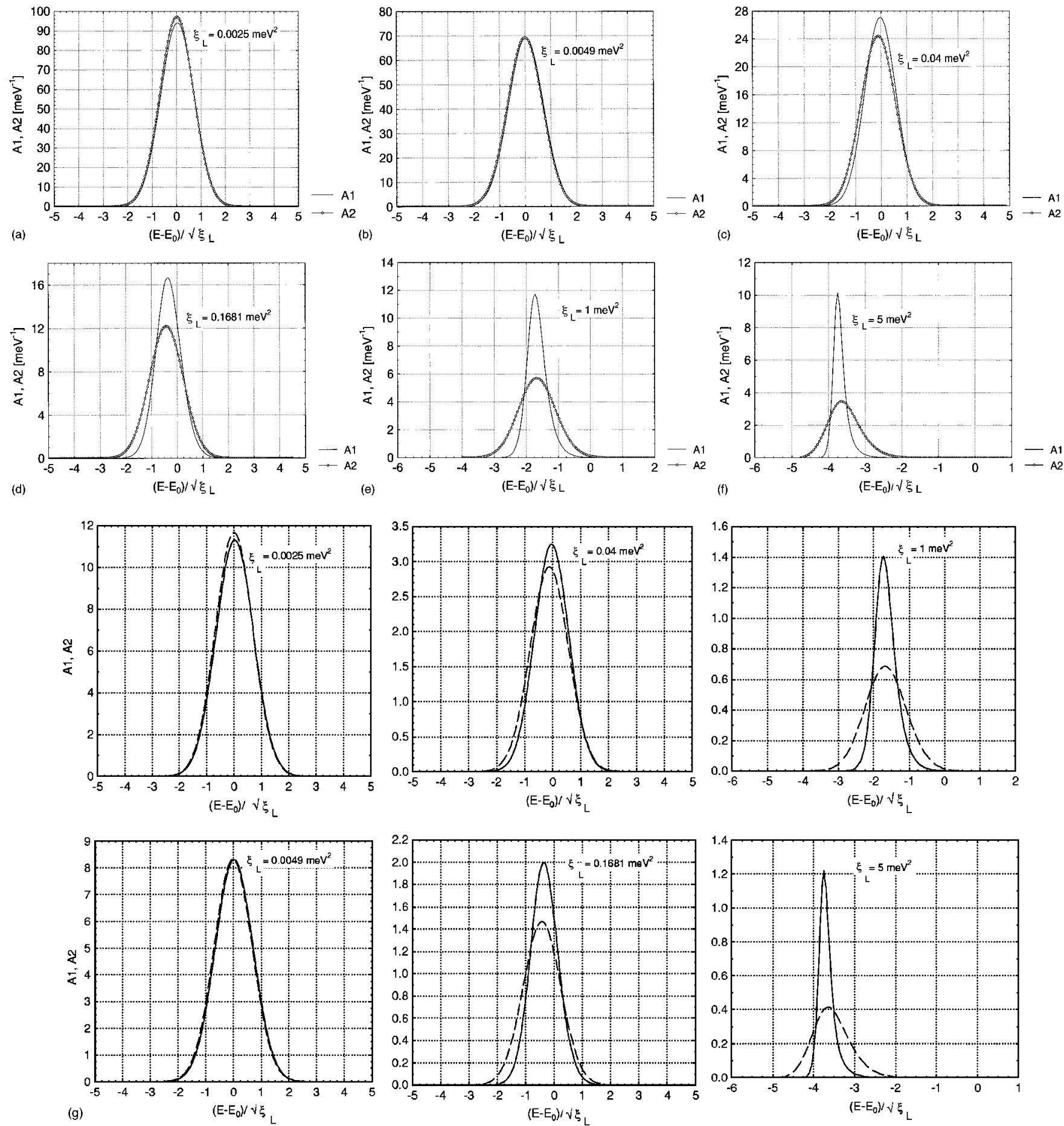

FIG. 5. Plot of the optical density functions corresponding to case 1 (A1, solid line) and case 2 (A2, dashed line) as a function of the normalized energy with respect to the standard deviation of the random potential fluctuations at different values of the variance: $\xi_{L}$ $=0.0025,0.0049,0.04,0.1681,1,25 \mathrm{meV}^{2}$ (all the plots are at $E_{L}=0.12 \mathrm{meV}$ ).

In Figs. 2(a), 2(b), and 3(a) and 3(b) the 3D trace plots of the normalized optical density from Eq. (50) as a function of the dimensionless energy with varying disorder parameter are shown for the first and the second cases, respectively. The spectra have been multiplied by the correlation energy in order to render them dimensionless. The exciton absorption line influenced by the random potential in both cases exhibits (i) a distinct asymmetry of the high- and low-energy shoulders of the spectrum with respect to the peak value, (ii) monotonic broadening and decrease of the magnitude of the exciton peak with increasing disorder, and (iii) shift of the maximum to lower energies. The redshift is clearly seen in Figs. 4(a) and 4(b) (for the first and second cases, respectively) where the optical density function is plotted against the energy in meV normalized with respect to the rms value of the amplitude of the random potential fluctuations $\sqrt{\xi_{L}}$ while the disorder parameter $\xi_{L}$ is varied. On increasing the standard deviation of the random potential fluctuations about the mean, the exciton absorption line broadens, the intensity of the spectrum decreases, and the peak shifts to lower ener- 
gies. The observed low-energy shift of the exciton peak with the disorder parameter (representing a measure of the depth of the random potential fluctuations) results from the fact that an increasingly greater part of the excitons becomes localized in the minima of the potential relief with an energy below the unperturbed band edge. The maximum of the excitonic energy distribution is centered at an energy corresponding to the exciton absorption peak. These results are consistent with previously calculated absorption spectra, e.g., the numerical calculation using the Green's function expansion applied in the one-dimensional case, ${ }^{17}$ and with results of Zimmermann ${ }^{9}$ and linear response theory results. ${ }^{20}$ From the figures it can be clearly seen that on reducing the disorder parameter the shape of the spectral line approaches a $\delta$-like free-exciton peak (in agreement with the expected $\delta$-shaped free-exciton spectrum). This confirms the ability of the method to correctly obtain the free-exciton limit at zero disorder. Another feature of the calculated optical density spectrum is the sharp decrease of its intensity below $\xi^{\prime} \sim 1$ [see Figs. 2(a) and 3(a)], i.e., for standard deviations of the random potential energy about its mean value of the order of the correlation energy $E_{L}$. Above the correlation energy the decrease of the magnitude of the exciton's peak is negligible and it remains almost constant up to very high values of $\xi^{\prime}$.
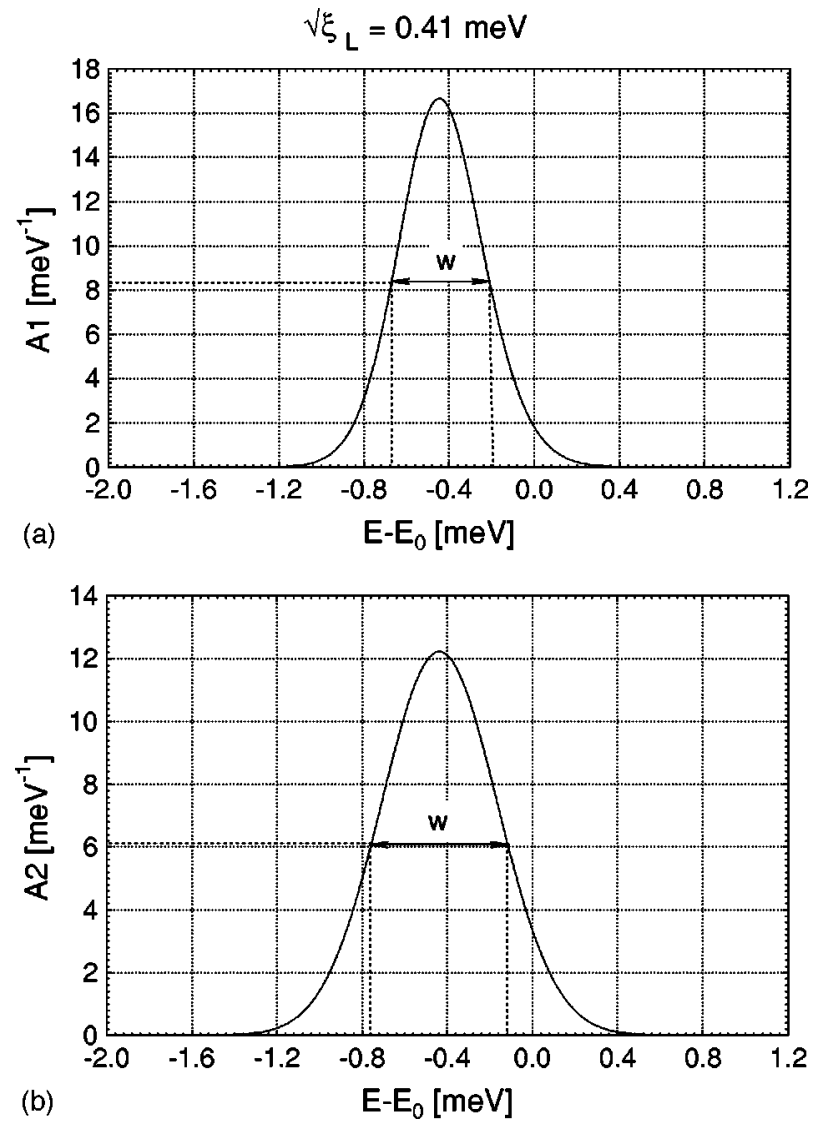

FIG. 6. (a) Optical density function calculated with the perturbation theory result for the high-energy tail and path-integral result for the low-energy tail of the spectrum (corresponding to case 1) vs energy at $\sqrt{\xi_{L}}=0.41 \mathrm{meV}$. The FWHM is denoted by $w\left(E_{L}\right.$ is kept constant at $0.12 \mathrm{meV}$ ). (b) Optical density function calculated with the path-integral result for both high- and low-energy tails of the spectrum (corresponding to case 2) vs energy at $\sqrt{\xi_{L}}=0.41 \mathrm{meV}$. The FWHM is denoted by $w\left(E_{L}\right.$ is kept constant at $\left.0.12 \mathrm{meV}\right)$.
This behavior can be explained by smoothing of the potential fluctuations with a characteristic length greater than the correlation length $L$.

In order to confirm the limiting behavior of the optical absorption spectrum with the perturbation theory results for weak disorder, we have plotted both high-energy spectra with the same path-integral low-energy side in Fig. 5 at different values of the disorder parameter (variance of the random potential fluctuations) $\xi_{L}$. As can be seen there exists a range of disorder up to $\sim 0.05 \mathrm{meV}^{2}$ where both approaches tend to the same high-energy tail; thus we can establish the limit of applicability of perturbation theory. Above this value the perturbations cannot be considered as small and the perturbation theory ceases to be valid.

In order to evaluate typical values of the full width at half maximum (FWHM) inferred from the calculated spectra we have taken the heavy-hole standard deviation and correlation energy values, namely, $\sqrt{\xi_{L}}=0.41$ and $E_{L}=0.12 \mathrm{meV}$, obtained in Ref. 7 by fitting the time-resolved photoluminescence data of Wang et al. ${ }^{10}$ The calculated optical absorption spectra are shown for two values of the variance, $\sqrt{\xi_{L}}$ $=0.41$ and $1 \mathrm{meV}$ in Figs. 6(a) and 6(b) and 7(a) and 7(b) (corresponding to the first and second cases considered, respectively). The problem of determining the energy zero of
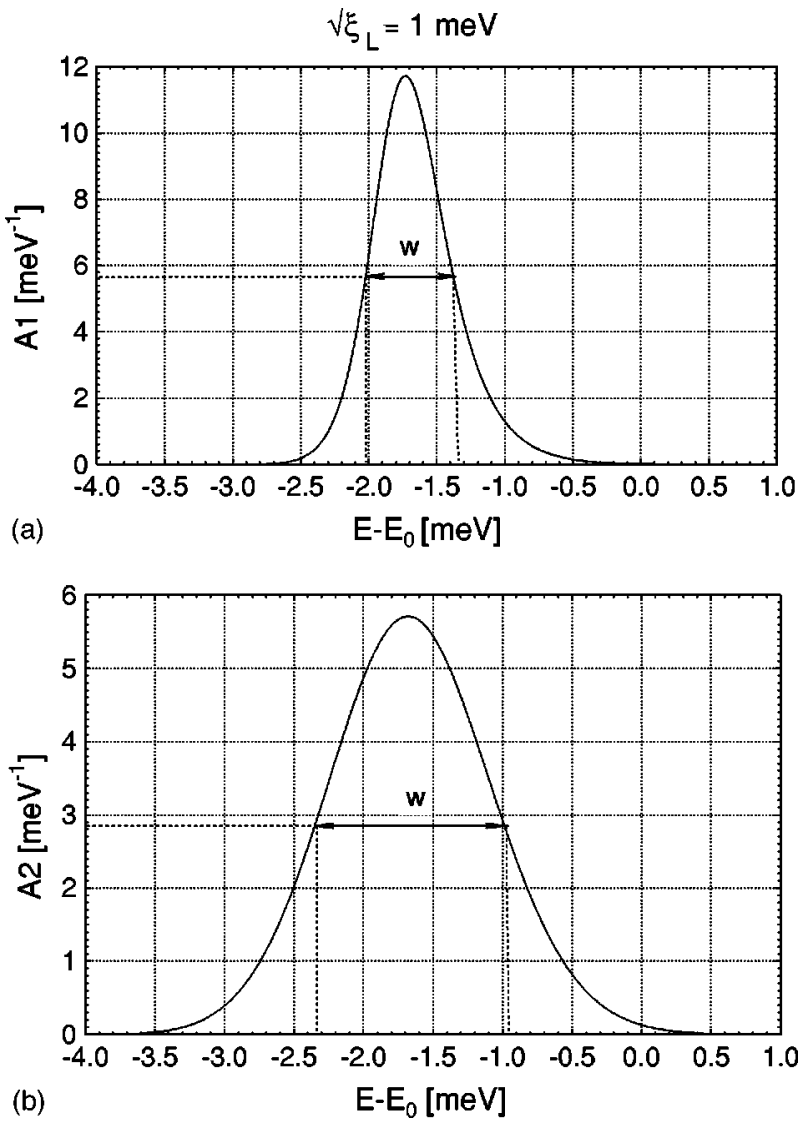

FIG. 7. (a) Optical density function calculated with the perturbation theory result for the high- energy tail and path-integral result for the low-energy tail of the spectrum (corresponding to case 1) vs energy at $\sqrt{\xi_{L}}=1 \mathrm{meV}$ (at a constant correlation energy $E_{L}$ $=0.12 \mathrm{meV}$ ). (b) Optical density function calculated with the pathintegral result for both high- and low-energy tails of the spectrum (corresponding to case 2) vs energy at $\sqrt{\xi_{L}}=1 \mathrm{meV}$ (at a constant correlation energy $E_{L}=0.12 \mathrm{meV}$ ). 


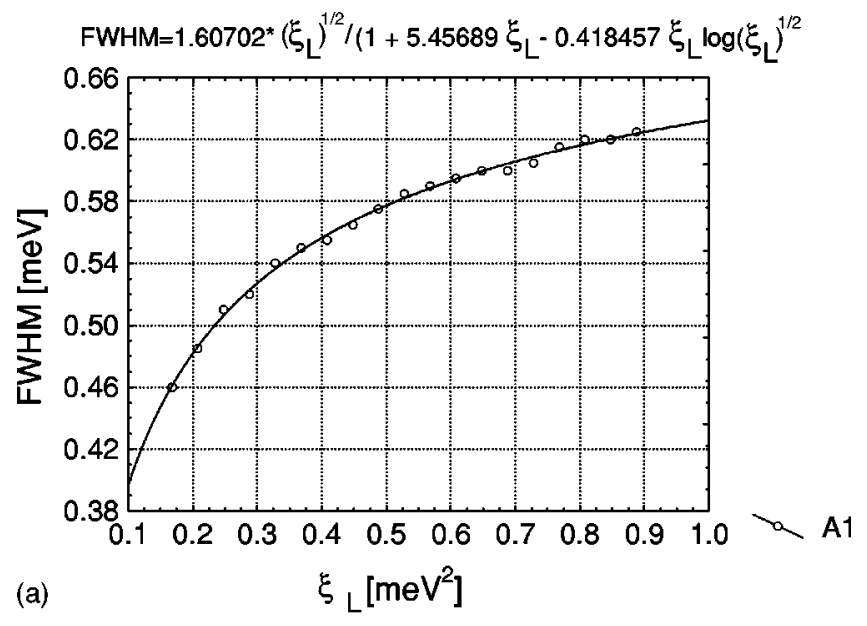

$\mathrm{FWHM}=1.60059\left(\xi_{L}\right)^{1 / 2} /\left(1+0.326992 \xi_{L}+0.025248 \xi_{L} \log \left(\xi_{L}\right)^{1 / 2}\right.$

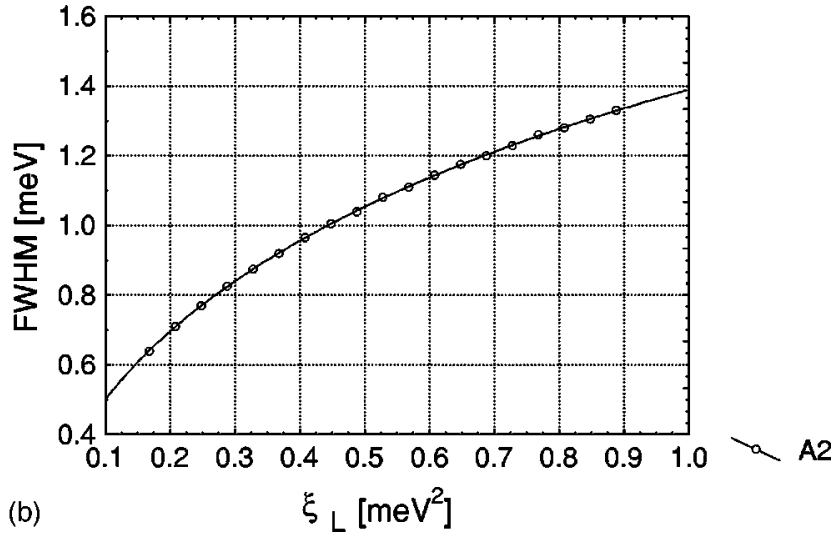

FIG. 8. Exciton absorption line width (FWHM) in meV vs variance of the random potential $\xi_{L}$ for the first (a) and second cases (b) described in the text. The solid line represents a fit performed with the function derived by forcing the spectral density Eq. (32) to a Gaussian, taking the FWHM as the standard deviation (the exact values of the coefficients are shown at the top of the graph; $x=\xi_{L}$ ).

the spectrum is not a trivial one, as has been pointed out by Efros Wetzel, ${ }^{2}$ but it has been shown to have an acceptable solution by Thouless and Elzain. ${ }^{36}$ In order to determine the energy zero, we have calculated the energy-zero shift due to the disorder according to the expression [Eq. (9)] derived in the latter, which is

$$
E_{0}^{*}=E_{0}-\frac{w^{2}}{4 \pi V}\left[1+\ln \left(\frac{128 \pi V^{2}}{w^{2}}\right)\right],
$$

where in the $2 \mathrm{D}$ case it has been shown ${ }^{30,37}$ that the energy zero of the path-integral DOS occurs at $E_{0}=-4 \mathrm{~V}$. In calculating the energy-zero shift, we have taken into account the previously established relation between the disorder parameters introduced within the tight-binding model and the corresponding parameters in the path-integral approach, ${ }^{30,37}$ namely, the tight-binding matrix element, representing the bandwidth $V=E_{L}$, and the variance $w^{2}=\xi_{L}$. The lowenergy shift of the peak can be clearly seen, as well as the shift of the maximum to the left with increasing standard deviation (from $\sqrt{\xi_{L}}=0.41$ to $1 \mathrm{meV}$ ) of the random potential fluctuations. (a)
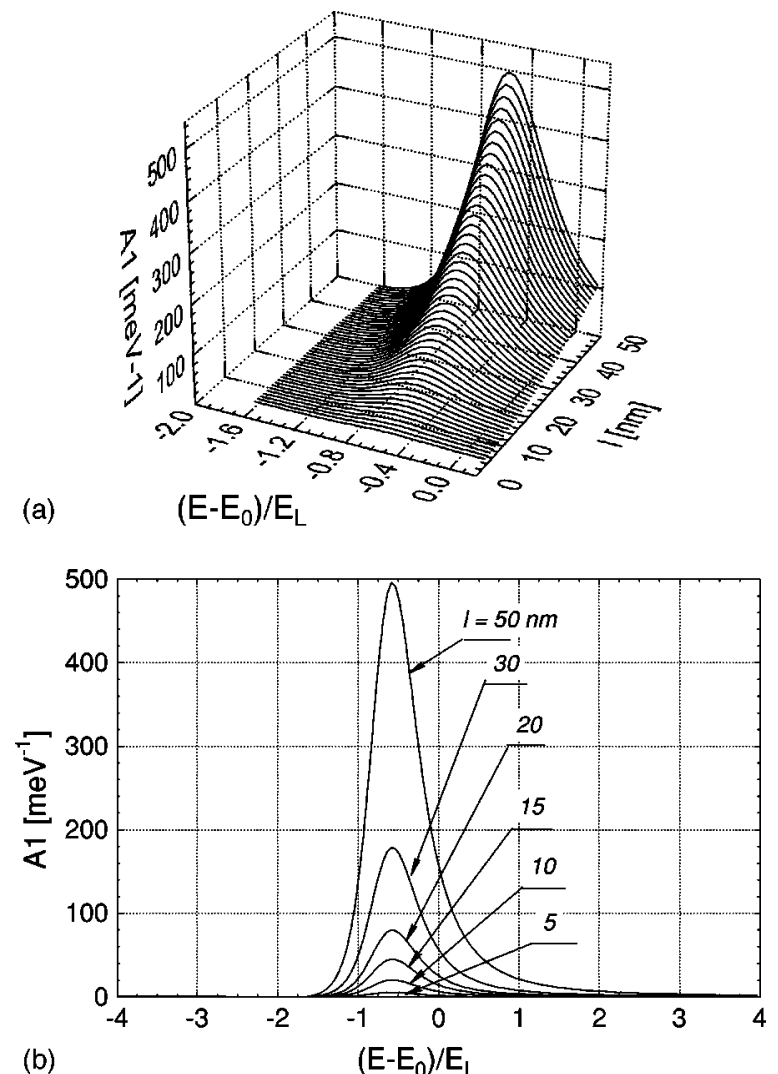

FIG. 9. (a) 3D plot of the optical density function corresponding to case 1 vs the energy, normalized with respect to the exciton correlation energy $E_{L}$ and the correlation length $l$ of the individual potentials. (b) Cross sections of (a): plots of the optical density function vs normalized energy corresponding to case 1 for $l$ $=5,10,15,20,50 \mathrm{~nm}$.

The exciton linewidths calculated from these figures are $w=0.46$ and $0.62 \mathrm{meV}$ for the first case and $w=0.63$ and $1.37 \mathrm{meV}$ for the second case. In order to evaluate the asymmetry of the calculated spectra, it is convenient to define high- and low-energy linewidths $w_{\mathrm{HE}}$ and $w_{\mathrm{LE}}$. The highenergy linewidths from Figs. 6(a) and 7(a) are 0.235 and $0.32 \mathrm{meV}$, while the low-energy linewidths are correspondingly 0.225 and $0.3 \mathrm{meV}$. Similarly, the high-energy linewidths inferred from Figs. 6(b) and 7(b) are 0.33 and 0.7 $\mathrm{meV}$ and the low-energy linewidths 0.3 and $0.67 \mathrm{meV}$. For both cases considered the high-energy linewidth is greater than the low-energy one, reflecting the steeper decay of the low-energy tail of the spectrum.

The exciton linewidths corresponding to the full pathintegral derivation (case 2) seem to be in better agreement with the reported experimental widths than the ones with a high-energy tail calculated using the perturbation theory (case 1). We have compared the FWHM obtained at $\sqrt{\xi_{L}}$ $=0.41 \mathrm{meV}$ (Fig. 6) with the exciton absorption linewidth of a single quantum well at $\sqrt{\xi_{L}}=0.4 \mathrm{meV}$ calculated in Ref. 5 (see Fig. 2). It should be noted that the second-case width of $0.63 \mathrm{meV}$ is closer to the value $w=0.75 \mathrm{meV}$ obtained from Fig. 2 of Ref. 5. The observed agreement of the pathintegral-inferred optical density spectra with the experimental data and up-to-date theoretical models can be attributed to the additional contribution of the localized exciton states 

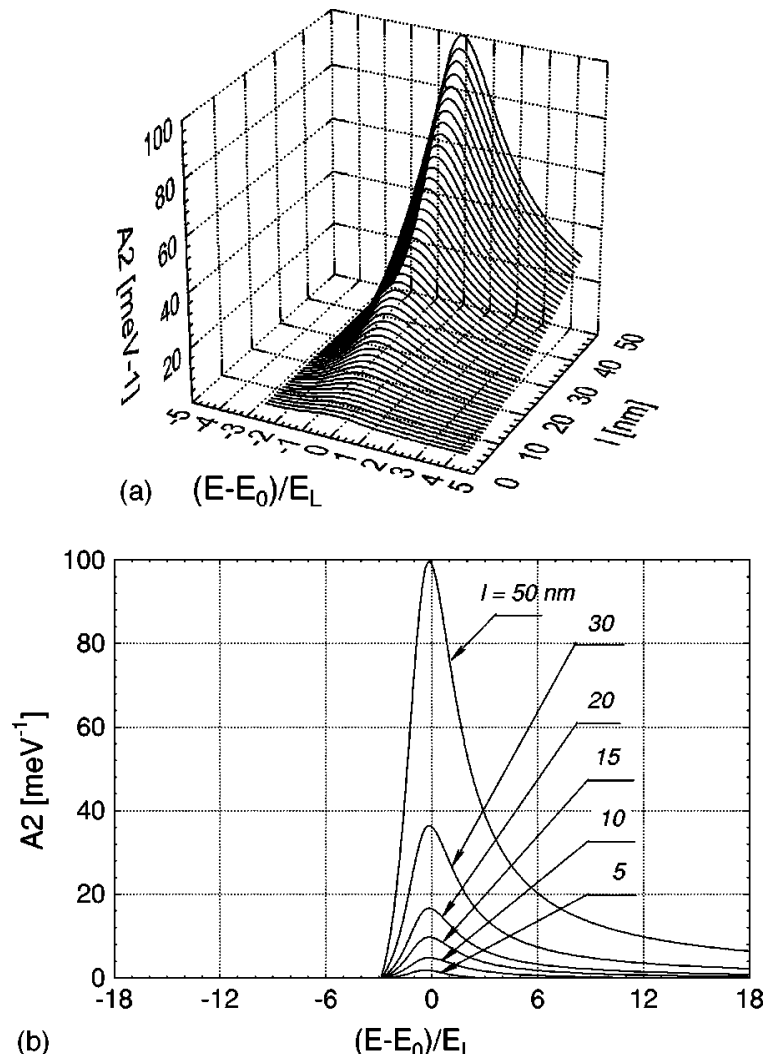

FIG. 10. (a) 3D plot of the optical density function corresponding to case 2 vs the energy, normalized with respect to the exciton correlation energy $E_{L}$ and the correlation length $l$. (b) Cross sections of (a): plots of the optical density function vs normalized energy corresponding to case 2 for $l=5,10,15,20,50 \mathrm{~nm}$.

from the Kane band tail, while in the perturbation theory method only the contribution of the completely delocalized states is considered.

In Figs. 8(a) and 8(b) we have plotted the exciton absorption linewidth (FWHM) as a function of the variance of the random potential $\xi_{L}$, varying within the interval $(0,1)$ for the first and second cases (the solid lines represent a fit to the data points). Both linewidths monotonically increase with increasing disorder and the relative broadening corresponding to the second case is greater than that for the first case. The best fit to the data is achieved using a function derived by forcing the spectral function from Eq. (32) to be Gaussian with standard deviation given by the FWHM. The FWHM in both cases depends to first order on the standard deviation $\sqrt{\xi_{L}}$ of the random potential, with higher-order terms giving rise to the asymmetric linewidth. Therefore instead of a normal (Gaussian) distribution of the spectral widths, characterized by a FWHM of $\sqrt{2 \xi_{L}}$, we have obtained additional terms responsible for the asymmetric shape of the spectral function.

In our calculations (Sec. II) we introduced essentially two disorder parameters, namely, the variance of the random potential $\xi_{L}$ and the correlation energy $E_{L}$. In order to study the effect of changing the correlation length of the potential fluctuations on the optical absorption spectrum we have fixed the variance at a value of $\sqrt{\xi_{L}}=0.5 \mathrm{meV}$, varying only the correlation energy (or equivalently the correlation length). The interval of variation of the correlation lengths has been chosen in conformity with the value for the correlation energy $E_{L}=0.12 \mathrm{meV}$ of Zimmermann, ${ }^{7}$ which gives $L$ $\approx 9.61 \mathrm{~nm}$, i.e., $L$ varies from 7 to $70 \mathrm{~nm}$. The calculated optical density functions are presented in Figs. 9(a) and 9(b) and 10(a) and 10(b). In each figure represents a 3D plot (a) and (b) the corresponding 2D sections at different values of the correlation length; Figs. 9 and 10 are for the first and second cases under consideration, respectively. We have introduced the new variable $l$, according to $L=l \sqrt{2}$, where $L$ is given in nanometers. It can be seen (Figs. 9 and 10) that increasing the correlation length (or equivalently $l$ ) and approaching the classical limit (i.e., $L \rightarrow \infty$ corresponding to perfect interface), the optical absorption spectrum tends to the free-exciton $\delta$ peak. In the opposite limit (decreasing the correlation length) continuous broadening of the exciton linewidth is observed, with a decrease in the peak magnitude and a low-energy shift of the maximum. In this case we approach the quantum case, which corresponds to the whiteGaussian-noise potential, when exciton localization takes place, resulting in the low-energy shift of the exciton peak. It should be noted, however, that these results are in contrast with the 2D optical absorption spectra calculated using linear optical susceptibility theory, shown in Fig. 6 of Ref. 18. where reduction of the correlation length reduces the FWHM and increases the magnitude of the peak.

To summarize, we believe that the classical limit of the optical density function can be reached in two equivalent ways, namely, either when the correlation length of the random potential fluctuations tends to infinity at a fixed depth of the potential fluctuations (as shown in Figs. 9 and 10) or by decreasing the magnitude (depth) of the potential fluctuations via $\xi_{L}$ while keeping the correlation energy (or equivalently $L$ ) constant (as, e.g., in Fig. 5). In both cases we obtain as a limiting behavior at zero disorder the free-exciton $\delta$ peak of the optical absorption, since when the perturbation potential is switched off the exciton should move freely. Therefore we expect that on approaching the classical limit the exciton line shape will become more and more symmetric Lorentzian, which at zero disorder should reproduce the $\delta$ peak of freeexciton absorption. The opposite quantum limit is reached either when $L \rightarrow 0$, at a fixed depth $\xi_{L}$ of the random potential fluctuations, or equivalently for energies deep in the excitonic band tail at a fixed correlation length $L$. In the first case the width of the typical potential well becomes increasingly smaller, while in the second case the potential well becomes deeper and steeper. Therefore increasing the disorder causes exciton localization to become more and more significant deep in the excitonic band tail, thus giving rise to Gaussian-Lorentzian-type asymmetric low-energy tails in the optical density.

\section{SUMMARY}

In this paper we have developed a semianalytical quantum-mechanical description of the optical absorption spectrum for $2 \mathrm{D}$ excitons in a rough $\mathrm{QW}$, taking into account exciton localization in the random potential fluctuations at the interface. The proposed method is based on the pathintegral technique for calculating density of states in disordered systems. In this model the exciton c.m. motion near the interface in the field of the random individual scattering po- 
tentials generated by local well-thickness fluctuations is considered. Gaussian statistics for the random distribution of the fluctuation potential is assumed. Asymptotic expressions for the low-and high-energy tails of the optical density function are obtained, and an alternative high-energy-tail asymptotic using the perturbation theory, limiting the absorption spectrum behavior at high energies and weak disorder, is also presented. Within the path-integral formalism two disorder parameters (characteristic energies) have been introduced, namely, the variance of the random potential fluctuations, representing a measure of the depth of the fluctuations, and the correlation energy, representing a measure of the exciton confinement. By using a nonlinear fitting procedure we have found an analytical interpolation function joining the two limiting asymptotics (for both cases under consideration) for any value of the variance of the random potential.

The calculated spectra exhibit the typical features observed in other methods of optical absorption calculations, such as the pronounced asymmetric shape, broadening of the excitonic line, and apparent low-energy shift of the maximum. Using the fitting parameters to the time-resolved experiments obtained in Ref. 7, a comparison has been made between the FWHM inferred from fully path-integral calculations and the calculations using perturbation theory for the high-energy tail. The comparison shows a much broader spectrum at strong enough disorder resulting from the fully path-integral approach than from the perturbation theory spectrum. This might be interpreted as due to the contribution of the localized exciton states in the high-energy tail of the spectrum, resulting from the semiclassical Kane tail in the density of states, which is absent within the perturbation theory approach, since only the free-exciton states are taken into account there. In both cases under consideration, we have found the leading term in the FWHM dependence on $\xi_{L}$ to be proportional to the standard deviation of the random potential plus correction terms responsible for the asymmetric line shape.

We have also studied the effect of varying the correlation length of the random potential fluctuations on the optical density function. Our results are consistent in both classical and quantum limits, tending to the free-exciton peak at large correlation lengths and monotonically broadening in the quantum limit. The proposed method of calculation is relatively simple, since it uses analytical expressions for the optical density function across the whole energy range.

\section{ACKNOWLEDGMENTS}

We are indebted to Dr. J. Watling for a critical reading of the manuscript. The authors (V.S.) and (G.S.) gratefully acknowledge the Thailand Research Fund (TRF) for financial support.
${ }^{1}$ J. Humlicek, E. Schmidt, L. Bocanek, R. Svehla, and K. Ploog, Phys. Rev. B 48, 5241 (1993).

${ }^{2}$ Al. Efros and C. Wetzel, Phys. Rev. B 52, 8384 (1995).

${ }^{3}$ D. S. Citrin, Phys. Rev. B 47, 3832 (1993).

${ }^{4}$ F. Yang, M. Wilkinson, E. J. Austin, and K. P. O’Donnell, Phys. Rev. Lett. 70, 323 (1993).

${ }^{5}$ L. C. Andreani, G. Panzarini, A. Kavokin, and M. Vladimirova, Phys. Rev. B 57, 4670 (1998).

${ }^{6}$ V. I. Belitsky, A. Cantarero, S. T. Pavlov, M. Gurioli, F. Bogani, A. Vinattieri, and M. Colocci, Phys. Rev. B 52, 16665 (1995).

${ }^{7}$ R. Zimmermann, Nuovo Cimento D 17, 1801 (1995).

${ }^{8}$ D. S. Citrin, Phys. Rev. B 54, 14572 (1996).

${ }^{9}$ R. Zimmermann, Phys. Status Solidi B 173, 129 (1992).

${ }^{10}$ H. Wang, J. Shah, T. C. Damen, and L. N. Pfeiffer, Phys. Rev. Lett. 74, 3065 (1995).

${ }^{11}$ S. Haacke, R. A. Taylor, R. Zimmermann, I. Bar-Joseph, and B. Deveaud, Phys. Rev. Lett. 78, 2228 (1997).

${ }^{12}$ J. Christen, M. Grundmann, and D. Bimberg, Appl. Surf. Sci. 41/42, 329 (1989).

${ }^{13}$ H. Castella and J. W. Wilkins, Phys. Rev. B 58, 16186 (1998).

${ }^{14}$ F. Yang, M. Wilkinson, E. J. Austin, and K. P. O’Donnell, Phys. Rev. Lett. 70, 323 (1993).

${ }^{15}$ M. Wilkinson, F. Yang, E. J. Austin, and K. P. O'Donnell, J. Phys.: Condens. Matter 4, 8863 (1992).

${ }^{16}$ B. I. Halperin, Phys. Rev. 139, A104 (1965).

${ }^{17}$ S. Glutsch and F. Bechstedt, Phys. Rev. B 50, 7733 (1994).

${ }^{18}$ S. Glutsch, D. S. Chemla, and F. Bechstedt, Phys. Rev. B 54, 11592 (1996).

${ }^{19}$ F. J. Dyson, Phys. Rev. 92, 1331 (1953).

${ }^{20}$ T. Strouken, C. Anthony, A. Knorr, P. Thomas, and S. W. Koch, Phys. Status Solidi B 188, 539 (1995).
${ }^{21}$ M. E. Raikh and Al. L. Efros, Fiz. Tverd. Tela (Leningrad) 25, 353 (1983) [ Sov. Phys. Solid State 25, 199 (1983)]

${ }^{22}$ E. Brezin and G. Parisi, J. Phys. C 13, L307 (1980).

${ }^{23}$ V. Samathiyakanit, J. Phys. C 7, 2849 (1974).

${ }^{24}$ V. Sa-yakanit, J. Phys. C 11, L521 (1978).

${ }^{25}$ V. Sa-yakanit and H. R. Glyde, in Path Summation: Achievement and Goals, Proceedings of the Adriatico Research Conference on "Path Integral Method with Application," Trieste, 1987, edited by S. Lundqvist, A. Ranfaagni, V. Sa-yakanit, and L. S. Schulman (World Scientific, Singapore, 1998).

${ }^{26}$ Piet Van Mieghem, Rev. Mod. Phys. 64, 755 (1992).

${ }^{27}$ G. Bastard, Wave Mechanics of Semiconductor Heterostructures (Les Editions de Physique, Les Ulis, France, 1988), p. 133.

${ }^{28}$ A. Efros and M. Raikh, in Optical Properties of Mixed Crystals, edited by R. J. Elliott and I. P. Ipatova, Vol. 23 of Modern Problems in Condensed Matter Sciences, edited by V. M. Agranovich, A. A. Maradudin, R. J. Elliott, and I. P. Ipatova (NorthHolland, Amsterdam, 1998).

${ }^{29}$ B. I. Halperin and M. Lax, Phys. Rev. 148, 722 (1966).

${ }^{30}$ V. Sa-yakanit, and G. Slavcheva, Phys. Rev. B 58, 13734 (1998).

${ }^{31}$ D. N. Quang and N. H. Tung, Phys. Status Solidi B 209, 375 (1998).

${ }^{32}$ V. Sa-yakanit and H. R. Glyde, Phys. Rev. B 22, 6222 (1980).

${ }^{33}$ V. Sa-yakanit, Phys. Rev. B 19, 2266 (1979).

${ }^{34}$ W. Sritrakool, V. Sa-yakanit, and H. R. Glyde, Phys. Rev. B 32, 1090 (1985).

${ }^{35}$ P. Van Mieghem, G. Borghs, and R. Mertens, Phys. Rev. B 44, 12822 (1991).

${ }^{36}$ D. J. Thouless and M. E. Elzain, J. Phys. C 11, 3425 (1978).

${ }^{37}$ V. Sa-yakanit, Phys. Lett. A 240, 167 (1998).

${ }^{38}$ K. Müller, B. Mehlig, F. Milde, and M. Schreiber, Phys. Rev. Lett. 78, 215 (1997). 